\title{
Prediction of the gasification kinetics of a single wood char particle from a limited set of parameters
}

\author{
G. Teixeira ${ }^{\mathrm{a}, *}$, L. Van de steene ${ }^{\mathrm{b}, 1}$, A. Ponthieux ${ }^{\mathrm{a}}$, S. Salvador ${ }^{\mathrm{c}, 2}$ \\ ${ }^{a}$ VERI-VEOLIA, Zone Portuaire, 291 Av. Dreyfous Ducas, 78520 Limay, France \\ ${ }^{\mathrm{b}}$ CIRAD-PERSYST, TA B114/16, 73 Rue JF Breton, 34398 Montpellier Cedex 5, France \\ ${ }^{\mathrm{c}}$ RAPSODEE, Mines - Albi, UMR 5302 CNRS, Route de Teillet, 81013 Albi CT Cedex 09, France
}

H I G H L I G H T S

- Three functions were built to calculate particle conversion rate of steam gasification, Boudouard reaction and combustion.

- Function determination includes the use of a validated particle model and a numerical experimental design.

- The functions take into account all phenomena controlling particle conversion rate of a char particle under a reactive atmosphere.

- The functions depend on a limited set of operating conditions - T, $p_{i}$ - and particle properties: $\varepsilon_{0}, e_{p, 0}$ and $A_{i}$.

- The functions can be easily adapted to a new wood char by confronting 3-5 experimental results to the function.

\author{
Keywords: \\ Gasification \\ Particle conversion rate \\ Char particle
}

Modeling

\section{A B S T R A C T}

Many biomass gasification models have been developed at reactor scale with the objectives of understanding the phenomena involved and optimizing the processes. Most of usual models consist in solving equations of heat, mass and momentum balance in a multiphase media. These equations call for source terms that provide production or consumption of heat and species by the reactions. The heat and mass source terms are directly derived from the particle conversion rate, $d X / d t$. This paper focuses on the determination of these source terms regarding the char gasification stage of the gasification process.

We propose three simple functions to calculate wood particle conversion rate of steam gasification, Boudouard reaction and combustion. The determination of each function is based on a validated char gasification model at particle size and a numerical experimental design. The model of char particle gasification includes all the complexity of the transformation: intrinsic reaction kinetic and both internal and external heat/mass transfer limitations. The experimental design was used to define simulations to be carried out, and to establish a relevant correlation between the particle conversion rate and a limiting set of 5 influencing parameters. Two of them are representative of the operating conditions - temperature and partial pressure of the reactant gas, the three others corresponds to particle properties: porosity, thickness, and reactivity constant.

The three functions were determined for the gasification of a wood chips char particle in a $\mathrm{H}_{2} \mathrm{O} / \mathrm{N}_{2}, \mathrm{CO}_{2} /$ $\mathrm{N}_{2}$, and $\mathrm{O}_{2} / \mathrm{N}_{2}$ atmosphere respectively. And finally, considering wood pellet char, we showed that the functions could be adapted to this new char from only 3 to 5 experiments, showing a good correspondence between prediction functions and experimental results.

\footnotetext{
* Corresponding author.

E-mail addresses: gabriel.teixeira@veolia.com (G. Teixeira), steene@cirad.fr (L. Van de steene), arnaud.ponthieux@veolia.com (A. Ponthieux), salvador@mines-albi.fr (S. Salvador).

1 Tel.: +33 4676165 22; fax: +33 467616515 .

2 Tel.: +335634930 26; fax: +33563493243.
} 


\section{Introduction}

Gasification has been shown to have a high potential for biomass energy production. Various forms of energy such as electricity, heat, or biofuels can be produced depending on the nature of the feedstock and the technology. Different types of technologies including fluidized or fixed bed, entrained flow, in single or staged reactors are now available on the market. Nevertheless, the economic competitiveness of these technologies remains questionable and efforts are required to improve the efficiency and reliability of the processes, and the flexibility of the feedstock. Modeling can help develop new designs for reactors as well as optimize existing ones.

Whatever the technology used, gasification reactors involve a successive or simultaneous series of thermal processes, mainly biomass drying and pyrolysis, oxidation/cracking of pyrolysis gases, and char gasification. Achieving complete carbon conversion is mainly controlled by the heterogeneous reactions that occur between the char produced during the pyrolysis stage, and the reacting gases such as $\mathrm{H}_{2} \mathrm{O}, \mathrm{CO}_{2}$ and $\mathrm{O}_{2}$ :

$\mathrm{C}+\mathrm{H}_{2} \mathrm{O} \rightarrow \mathrm{CO}+\mathrm{H}_{2} \quad$ Steam gasification

$\mathrm{C}+\mathrm{CO}_{2} \rightarrow 2 \mathrm{CO}$ Boudouard reaction

$\mathrm{C}+\mathrm{O}_{2} \rightarrow \mathrm{CO} / \mathrm{CO}_{2}$ Combustion

Numerous industrial or academic codes have been developed for fluidized bed, fixed bed or entrained flow reactors. These complex models consist in solving mass, energy, and momentum balance equations in the multi-phase media. Balanced equations require source terms that provide local production or consumption rates of species and heat. Regarding the heterogeneous reactions (Eqs. (1)-(3)), the mass/heat source terms can be calculated directly from the particle conversion rate of the char particles, as follows $\left(\frac{d m}{d t}\right)_{i}=\left(\frac{d X}{d t}\right)_{i} \cdot m_{0}$ and $Q_{i}=\left(\frac{d X}{d t}\right)_{i} \cdot m_{0} \cdot \Delta_{r, j}^{T}$ respectively, where $\left(\frac{d m}{d t}\right)_{i}$ and $Q_{i}$ are the mass and heat source terms of the reaction $i$ respectively.

$\left(\frac{d X}{d t}\right)_{i}$ is the particle conversion rate of the reaction $i$.

$X$ is the char conversion progress and depends on the mass at time $t, m_{t}$, the initial mass, $m_{0}$, and the mass of ashes, $m_{a s h}$, as follows: $X=\frac{m_{0}-m_{t}}{m_{0}-m_{a s h}}$.

Intrinsic reaction kinetics cannot be used to express the particle conversion rate as the dimension of the char particles is such that heat and mass transfers limit their conversion. Consequently, the particle conversion rate has to be calculated to take into account limitations to heat and mass transfer.

At the present time, two types of sub-models are commonly used to calculate these particle conversion rates:

- A complex sub-model of the gasification of a char particle that solves all the conservation equations at particle scale [1-3]. These sub-models allow a fine description of the physical and chemical phenomena at particle scale. Changes in particle size due to reactions and fragmentation can also be taken into account in the most sophisticated sub-model [4]. The main problem with this approach is that the process codes built in this way are too time consuming to be practical for optimization or design purposes.

- A simple sub-model is the most widely used solution to express the source terms [5-9]. It consists in expressing the particle conversion rate as a function of intrinsic reaction kinetics and diffusional resistances for external heat and mass transfers. This solution considerably simplifies char bed modeling as no description is required at particle scale. Nevertheless such theoretical formulations have not been validated for different particle sizes, operating conditions, and different types of biomass. Moreover, the formulations do not consider internal diffusion in the particle, as shrinking core models are usually used to model particle conversion progress.

To sum up, there is a need to define simple expressions for particle conversion rates for char gasification reactions (Eqs. (1)-(3)) that take into account the complexity of the transformation at particle scale: intrinsic reaction kinetics and both internal and external heat/mass transfer limitations. This paper proposes three correlations to express particle conversion rates for the three reactions involved in char gasification. These correlations are based on a limited set of parameters:

- operating conditions, i.e. temperature and partial pressure of the reacting gas;

- the nature of the biomass through the intrinsic reaction kinetics of the char and its initial porosity;

- particle size.

The functions to calculate particle conversion rates were developed for wood chips char (WCC). They were subsequently adapted for the gasification of a new char made from wood pellets (WPC) thanks to a few validation experiments.

Such correlations can be used in reactor models to determine source terms for char gasification reactions if WCC or WPC made from pine are being used.

\section{Methodology}

The methodology we developed to calculate the particle conversion rate involves the following steps:

- development and calibration of a detailed particle model that enables the prediction of the particle conversion rate of a char particle under various gasification operating conditions $[3,10]$;

- choice of a limited set of influencing parameters regarding gasification reactions;

- determination of functions thanks to "numerical" experiments using the validated model. 


\subsection{The particle model}

The correlations to determine the particle conversion rates are based on the use of an existing particle model previously developed by our team [10]. The model enables prediction of the gasification of a char particle in $\mathrm{N}_{2}$ atmospheres containing $\mathrm{H}_{2} \mathrm{O}, \mathrm{CO}_{2}$ and $\mathrm{O}_{2}$ following the reactions (Eqs. (1)-(3)).

Briefly, the model considers all main phenomena that control the particle conversion rate of a char particle under a reactive atmosphere, namely external and internal heat/mass transfers and intrinsic reaction kinetics. For a given set of operating conditions and char properties, the solution is given by the resolution of mass, energy, and momentum balance equation in a spherically symmetric, one dimensional mesh. Temperature and partial pressure of the reacting gas, and char properties such as porosity, permeability, tortuosity, are fixed at the start. The model calculates changes in physical values inside the particle during conversion. In the present work, the only output of interest from the model is the global conversion of the char particle versus time: $X(t)$. Accurate sensitivity of the calculated conversion to operating conditions and particle size was demonstrated by comparing simulations with experimental results [10].

An important point in the development of the model was the choice of kinetic model and the determination of the kinetic constants and the concentration of active sites. Whatever the reaction concerned (Eqs. (1)-(3)), char reactivity depends on temperature, gas partial pressure ( $n$-order dependence) and the concentration of active sites:

$R_{i}=K_{i}^{\text {int }} \cdot P_{i}^{n_{i}} \cdot F_{i}(X)$

where $p_{i}(\mathrm{~atm})$ is the partial pressure and $n_{i}(-)$ is the order of the reaction $i . K_{i}^{\text {int }}\left(\mathrm{s} \mathrm{atm}^{-n}\right)$ is the intrinsic reaction kinetics of the reactant gas $i$ :

$K_{i}^{\text {int }}=A_{i} \cdot \exp \left(\frac{-E_{i}}{R \cdot T}\right)$

$A_{i}\left(\mathrm{~s}^{-1} \mathrm{~atm}^{-n i}\right)$ and $E_{i}\left(\mathrm{~J} \mathrm{~mol}^{-1}\right)$, respectively the pre-exponential factor and the activation energy of the heterogeneous reaction $i$ were previously determined for each reaction, by comparing experimental and model results. $F_{i}(X)$, called surface function, was used to describe the change in the concentration of active sites during conversion. More generally, the surface function enables all the phenomena involved in the change in reactivity during gasification to be taken into account: the surface function was determined experimentally in a previous work [10].

The model was experimentally validated for wood chips char (WCC) in the following operating conditions:

- temperature between 1073 and $1323 \mathrm{~K}$;

- $\mathrm{H}_{2} \mathrm{O}$ partial pressure between 0.1 and $0.4 \mathrm{~atm}$;

- $\mathrm{CO}_{2}$ partial pressure between 0.1 and $0.4 \mathrm{~atm}$;

- $\mathrm{O}_{2}$ partial pressure between 0.03 and $0.12 \mathrm{~atm}$;

- char particle thickness between 1.5 and $6.5 \mathrm{~mm}$.

As an example, in the case of gasification of a char particle in $\mathrm{H}_{2} \mathrm{O} / \mathrm{N}_{2}$ atmosphere, model sensitivity to partial pressure (a), temperature (b), and char particle thickness (c) is shown in Fig. 1. Experimental results are also plotted in the figure to show the accuracy of the simulations.

These curves, which were discussed in detail in our previous paper [10] form the basis of the methodology proposed here to calculate the functions. Indeed, the particle conversion rate $\frac{d X}{d t}$ for a set of operating conditions is given by the slope of the corresponding curve in Fig. 1. It is noteworthy that whatever the set of operating conditions, the particle conversion rate remains constant until $90 \%$ of the char has been converted and decreases thereafter. From this
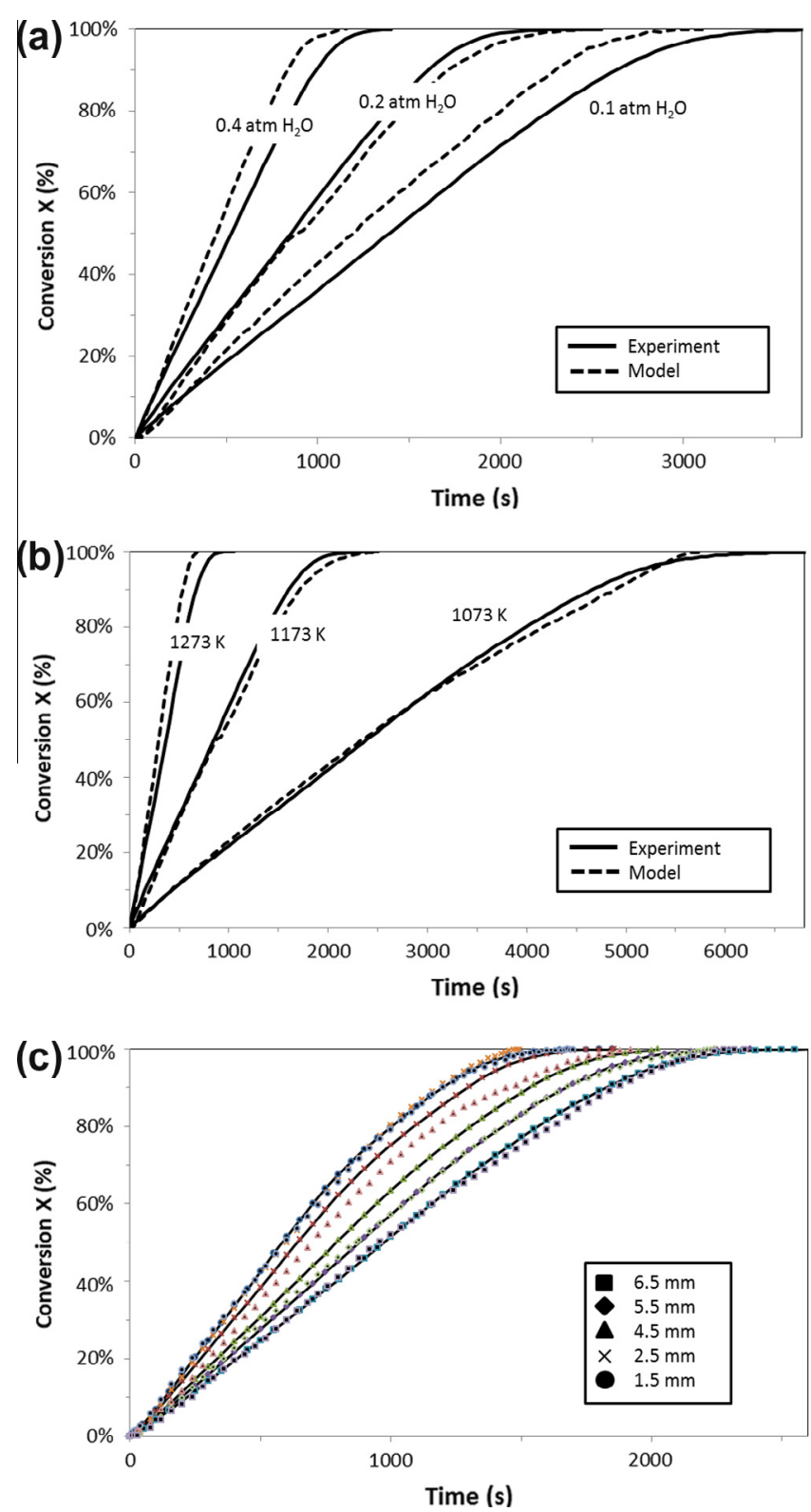

Fig. 1. Example of model results; gasification of a char particle in $\mathrm{H}_{2} \mathrm{O} / \mathrm{N}_{2}$ atmosphere at different partial pressures of reactant (a), temperatures (b), and char particle thicknesses (c) (experiment and model) referential operating conditions: temperature of bulk gas, $1173 \mathrm{~K}$; char thickness, $5.5 \mathrm{~mm}$; partial pressure of reactant gas, $0.2 \mathrm{~atm} \mathrm{H}_{2} \mathrm{O}$; porosity, 0.65 ; pre-exponential factors, $35.5 \times 10^{4} \mathrm{~s}^{-1} \mathrm{~atm}^{-0.8}[10]$.

observation and for the sake of simplicity, we decided to predict the particle conversion rate as a single value, independent of char conversion and equal to its value at $50 \%$ of conversion. The consequence is that our simplified particle conversion rate overestimates the reactivity at the end of the conversion, but nevertheless remains acceptable when considering the conversion as a whole. The description of the end of gasification remains a delicate and unsolved problem because fracturation, the catalytic effect of minerals, and the development of porosity all play a major role. This point, which requires more fundamental research, is beyond the scope of this paper, where our objective is establishing simple practical correlations.

Furthermore, developing three independent correlations for the three heterogeneous reactions implies that synergistic and antagonistic phenomena between reactions are not taken into consideration. Several authors have investigated the effect of inter- 
actions between various reactive gases on gasification reactivity, but their results remain questionable $[11,12]$. Nevertheless, in cases where interactions were observed, their impacts on the particle conversion rate were relatively weak. Consideration of these interactions was in any case not feasible in the simplified approach we proposed to determine the functions.

\subsection{Choice of influencing parameters}

The particle conversion rate of char gasification depends on many parameters that affect both intrinsic reaction kinetics and heat/mass transfer limitations. These parameters can be classified in two categories:

i. Parameters that affect operating conditions. This category includes the reaction temperature, the partial pressure of the reactant gas, and the gas flow velocity around the particle.

ii. Parameters that affect char particle properties. This category includes intrinsic reaction kinetics, porosity, particle thickness and mineral composition.

The choice of the parameters with the most influence on char particle conversion is a determining point of the methodology developed. Indeed, the number of parameters has to be high enough to allow the correlation to reproduce all the phenomena involved in char particle gasification. But for the sake of simplicity, the number of parameters has to be as small as possible. Our choice of parameters is now given and discussed.

- Regarding operating conditions.

Temperature and the partial pressure of the reactant gas were naturally chosen as their influence on intrinsic reaction kinetics is well known and clearly illustrated in Fig. $1 \mathrm{a}$ and $\mathrm{b}$ for $\mathrm{C}-\mathrm{H}_{2} \mathrm{O}$ gasification in our operating conditions.

Gas flow velocity was not taken into account because it was found to have a negligible impact on char particle gasification [13].

- Regarding char particle properties.

We chose the initial char particle porosity and thickness because of their influence on internal and external heat/mass transfers. The thickness of the WCC particle has a major impact on reactivity (Fig. 1c). In a previous study, we showed that the other dimensions do not influence particle conversion rate [10]. Sensitivity to char porosity was checked by performing simulations with porosity varying from 0.5 to 0.8 in typical operating conditions (see Fig. 2). Results clearly showed the sensitivity of particle conversion rate to this parameter. For the sake of simplicity, we thus chose to use the initial value of particle porosity and thickness.

To account for intrinsic reaction kinetics, we chose the preexponential factor Ai. Fig. 3 illustrates sensitivity to $A_{i}$ through simulations performed with $A_{i}$ varying between $2.1 \times 10^{5}$ and $5.4 \times 10^{5} \mathrm{~s}^{-1} \mathrm{~atm}^{-0.8}$. Regarding intrinsic reaction kinetics, we assume that sensitivity to temperature $\left(E_{a}\right)$ and partial pressure $(n)$ do not depend on the nature of the char. $E_{a}$ and $n$ are constant whatever the kind of char considered. Values are those obtained with WCC (Table 1).

Finally, we selected five parameters to calculate particle conversion rates of heterogeneous reactions. The three functions take the form:

$\left(\frac{d X}{d t}\right)_{i}=f\left(T, P_{i}, \varepsilon_{p, 0}, e_{p, 0}, A_{i}\right)$

where $i$ is the heterogeneous reaction subscript (steam gasification, the Boudouard or combustion reaction); $T(\mathrm{~K})$ and $p_{i}(\mathrm{~atm})$ are

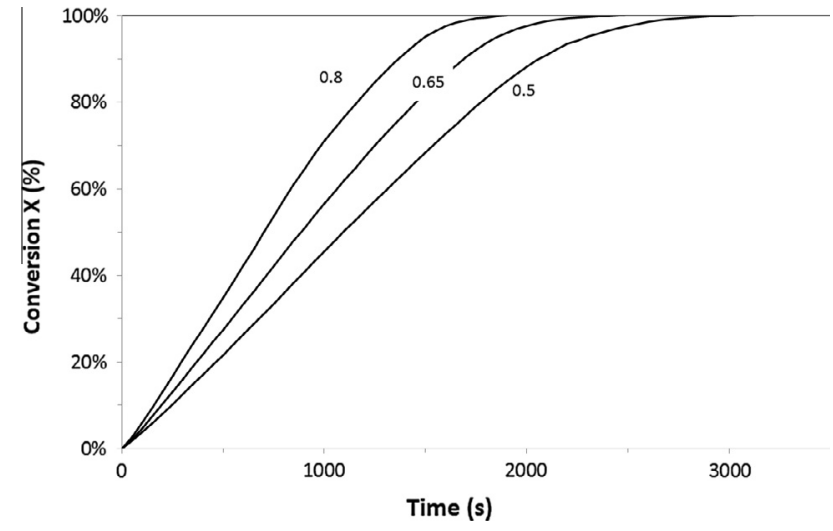

Fig. 2. Simulation results of WCC gasification for three char porosities: $0.5,0.65$ and 0.8 . Temperature of bulk gas: $1173 \mathrm{~K}$; char thickness: $5.5 \mathrm{~mm}$; partial pressure of reactant gas: $0.2 \mathrm{~atm} \mathrm{H}_{2} \mathrm{O}$, pre-exponential factors: $35.5 \times 10^{4} \mathrm{~s}^{-1} \mathrm{~atm}^{-0.8}$.

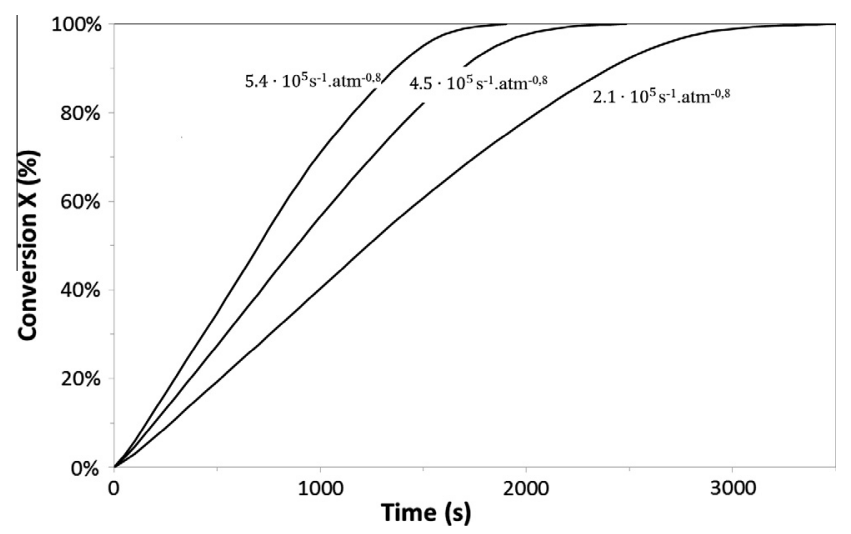

Fig. 3. Simulation results of WCC gasification for three pre-exponential factors: $2.1 \times 10^{5}, 4.5 \times 10^{5}$, and $5.4 \times 10^{5} \mathrm{~s}^{-1} \mathrm{~atm}^{-0.8}$. Temperature of bulk gas: $1173 \mathrm{~K}$; char thickness: $5.5 \mathrm{~mm}$; partial pressure of reactant gas: $0.2 \mathrm{~atm} \mathrm{H}_{2} \mathrm{O}$, porosity: 0.65 .

respectively the bulk temperature and the partial pressure of the reactant gases in the atmosphere; $\varepsilon_{p, 0}(-)$ and $e_{p, 0}(\mathrm{~m})$ are respectively, the initial porosity and thickness of the particle; $A_{i}\left(\mathrm{~s}^{-1} \mathrm{~atm}^{-n i}\right)$ is the pre-exponential factor of the reaction $i$.

\subsection{Statistical method for the determination of the functions}

The validated particle model was used to produce particle conversion rate values $\frac{d X}{d t}$ at $50 \%$ of conversion for various sets of parameters $\left(T, p_{i}, \varepsilon_{p, 0}, e_{p, 0}, A_{i}\right)$. Then, the function that fit all the simulation results best was determined using a statistical method. The aim of the method was also to guide us in the choice of the simulation to be performed to best capture the sensitivity of the particle conversion rate to the parameters concerned. In other words, we set up an experimental design. For this purpose the Modde 9.0 software, (Umetrics, Umeå, Sweden) was used to successively:

- set up an experimental design to determine the best simulations (numerical experiment) to be performed with the particle model;

- determine the "particle conversion rate" as a correlation between the particle conversion rate $\frac{d X}{d t}$ calculated with the particle model and the variables retained $\left(T, p_{i}, \varepsilon_{0}, e_{p, 0}, A_{i}\right)$ that minimize errors between the function and the results of the particle model;

- validate the function by comparing it with the results of the particle model. 
Table 1

Intrinsic kinetic parameters of the wood chips char (WCC) [10].

\begin{tabular}{llll}
\hline Factors & $\begin{array}{l}\text { Steam-gasification } \\
\text { reaction }\end{array}$ & Boudouard reaction & Oxidation reaction \\
\hline$A$ & $3.55 \times 10^{5} \mathrm{~s}^{-1} \mathrm{~atm}^{-0.8}$ & $1.2 \times 10^{8} \mathrm{~s}^{-1} \mathrm{~atm}^{-0.7}$ & $1.1 \times 10^{9} \mathrm{~s}^{-1} \mathrm{~atm}^{-0.6}$ \\
$E_{a}\left(\mathrm{~J} . \mathrm{mol}^{-1}\right)$ & $170 \times 10^{3}$ & $3245 \times 10^{3}$ & $17.94 \times 10^{3}$ \\
$n(-)$ & 0.8 & 0.7 & 0.6 \\
\hline
\end{tabular}

In the following, we present successively the chosen experimental designs and the development domain of the functions.

The experimental design was based on a first screening to validate our choice of influencing parameters in terms of the sensitivity of the particle conversion rate. It was also used to identify the most significant interactions between the parameters. Then a second screening was performed to quantify the variation in the response "particle conversion rate" with a variation in a given parameter. For readers familiar with experimental design, we performed a two level factorial experiment and a Latin hypercube design successively. These two experimental designs generated a numerical database to help determine the polynomial function. Function coefficients were calculated using the least squares method to minimize errors between the function and numerical experiments.

The development domain of one function is the set of parameter values for which the function is developed. This domain is characterized by a low limit and high limit for each parameter (Table 2).

Concerning temperature $T$ and partial pressure $p_{j}$, low and high limits correspond to the operating conditions that can be used in a continuous char gasification reactor [14]. For the Boudouard reaction, steam gasification, and combustion, low limits were fixed at $1173 \mathrm{~K}, 1073 \mathrm{~K}$ and $973 \mathrm{~K}$ respectively.

Concerning initial particle thickness $e_{p, 0}$, the lower limit was set at $1.5 \mathrm{~mm}$ because below this value particle thickness no longer affects particle conversion rate [15-18]. The upper limit was set at $6.5 \mathrm{~mm}$ based on the thickness distribution analysis found in [14]: $95 \%$ of the char particles were $0-6.5 \mathrm{~mm}$ thick.

Initial porosity $\varepsilon_{0}$ was determined from porosity measurement performed on WPC (0.80) and WCC (0.50) [14]. These values are taken as the upper and lower limits respectively.

The upper and lower limits of pre-exponential $A_{i}$ factor were calculated so as to vary the particle conversion rate in a range of $\pm 30 \%$ from the referential conditions. Referential gasifying conditions are $T=1173 \mathrm{~K}, p_{\mathrm{H} 2 \mathrm{O}}$ and $p_{\mathrm{CO} 2}=0.2 \mathrm{~atm}$, and $p_{\mathrm{O} 2}=0.03$. Referential properties of WWC particles are $e_{p, 0}=5.5 \times 10^{-3} \mathrm{~m}$,

Table 2

Range of variation for each parameters.

\begin{tabular}{llll}
\hline & Parameters & $\begin{array}{l}\text { Limit } \\
\text { Low }\end{array}$ & High \\
\hline Steam gasification reaction & $T(\mathrm{~K})$ & 1073 & 1373 \\
& $p_{\mathrm{H} 2 \mathrm{O}}(\mathrm{atm})$ & 0.1 & 0.4 \\
& $e_{p, 0}(\mathrm{~m})$ & $1.5 \times 10^{-3}$ & $6.5 \times 10^{-3}$ \\
& $\varepsilon_{p, 0}(-)$ & 0.5 & 0.8 \\
& $A_{\mathrm{H} 2 \mathrm{O}}\left(\mathrm{s}^{-1} \mathrm{~atm}^{-0.8}\right)$ & 210,000 & 540,000 \\
& $T(\mathrm{~K})$ & 1173 & 1373 \\
Boudouard reaction & $p_{\mathrm{CO} 2}(\mathrm{~atm})$ & 0.1 & 0.4 \\
& $e_{p, 0}(\mathrm{~m})$ & $1.5 \times 10^{-3}$ & $6.5 \times 10^{-3}$ \\
& $\varepsilon_{p, 0}(-)$ & 0.5 & 0.8 \\
& $A_{\mathrm{CO} 2}\left(\mathrm{~s}^{-1} \mathrm{~atm}^{-0.7}\right)$ & $0.75 \times 10^{8}$ & $16.5 \times 10^{8}$ \\
& $T(\mathrm{~K})$ & 973 & 1373 \\
Oxidation reaction & $p_{\mathrm{O} 2}\left(\mathrm{~atm}^{8}\right)$ & 0.03 & 0.12 \\
& $e_{p, 0}(\mathrm{~m})$ & $1.5 \times 10^{-3}$ & $6.5 \times 10^{-3}$ \\
& $\varepsilon_{p, 0}(-)$ & 0.5 & 0.8 \\
& $A_{\mathrm{O} 2}\left(\mathrm{~s}^{-1} \mathrm{~atm}^{-0.6}\right)$ & $0.1 \times 10^{9}$ & $8 \times 10^{9}$ \\
\hline
\end{tabular}

Table 3

Coefficient values of the functions for the three reactions.

\begin{tabular}{llll}
\hline Reaction & Steam gasification & Boudouard & Oxidation \\
\hline$a$ & $-2.38 \times 10^{1}$ & $-3.30 \times 10^{1}$ & -7.83 \\
$b$ & $2.92 \times 10^{-2}$ & $4.18 \times 10^{-2}$ & $8.65 \times 10^{-3}$ \\
$c$ & 2.63 & 2.86 & $2.32 \times 10^{1}$ \\
$d$ & $3.72 \times 10^{2}$ & $6.01 \times 10^{2}$ & $-2.89 \times 10^{2}$ \\
$e$ & -4.33 & -6.42 & -3.52 \\
$f$ & $3.32 \times 10^{-6}$ & $1.48 \times 10^{-8}$ & $1.13 \times 10^{-10}$ \\
$g$ & $-1.02 \times 10^{-5}$ & $-1.45 \times 10^{-5}$ & $-3.12 \times 10^{-6}$ \\
$h$ & -2.88 & -2.90 & $-6.59 \times 10^{1}$ \\
$i$ & $-4.08 \times 10^{-1}$ & $-5.86 \times 10^{-1}$ & $2.85 \times 10^{4}$ \\
$j$ & $3.39 \times 10^{-3}$ & $4.86 \times 10^{-3}$ & 3.78 \\
$k$ & $-2.59 \times 10^{-9}$ & $-1.12 \times 10^{-11}$ & $-4.95 \times 10^{-3}$ \\
$l$ & $8.38 \times 10^{-7}$ & $1.34 \times 10^{2}$ & $-9.54 \times 10^{-2}$ \\
$m$ & $1.19 \times 10^{2}$ & $-2.87 \times 10^{-7}$ & $-8.36 \times 10^{-14}$ \\
$n$ & $-8.17 \times 10^{-5}$ & $4.04 \times 10^{-9}$ & \\
$o$ & $1.05 \times 10^{-6}$ & & \\
\hline
\end{tabular}

$\varepsilon_{0}=0.75$ and $A_{\mathrm{H} 2 \mathrm{O}}=3.55 \times 10^{3} \mathrm{~s}^{-1} \mathrm{~atm}^{-0.8}, \quad A_{\mathrm{CO} 2}=1.2 \times 10^{8} \mathrm{~s}^{-1}$ $\mathrm{atm}^{-0.7}$ and $A_{\mathrm{O} 2}=1.1 \times 10^{9} \mathrm{~s}^{-1} \mathrm{~atm}^{-0.6}$.

\section{Functions for wood chip char}

To improve the quality of the regression, a variable transformation in decimal logarithmic was performed so that the functions take the form

$\log _{10}\left(\frac{d X}{d t}\right)=f\left(T, P_{i}, \varepsilon_{p, 0}, e_{p, 0}, A_{i}\right)$

Functions of the particle conversion rates in the three atmospheres are given in Eqs. (8)-(10), and the coefficient values are listed in Table 3

Values of the correlation coefficient $R^{2}$ and predictive coefficient $Q^{2}$, which reflect the descriptive and predictive quality, respectively, are listed in Table 4 . They are greater than 0.95 whatever the reaction considered, attesting to their good quality.

Steam gasification function:

$$
\begin{aligned}
\log _{10}\left(\frac{d X}{d t}\right)= & a+b \cdot T+c \cdot P_{\mathrm{H} 20}+d \cdot e_{p}+e \cdot \varepsilon_{p}+f \cdot A_{\mathrm{H} 2 \mathrm{O}} \\
& +g \cdot T^{2}+h \cdot P_{\mathrm{H} 2 \mathrm{O}}^{2}+i \cdot T \cdot e_{p}+j \cdot T \cdot \varepsilon_{p}+K \cdot T \cdot A_{\mathrm{H} 2 \mathrm{O}} \\
& +l \cdot P_{\mathrm{H} 2 \mathrm{O}} \cdot A_{\text {vapo }}+m \cdot e_{p} \cdot \varepsilon_{p}+n \cdot e_{p} \cdot A_{\mathrm{H} 2 \mathrm{O}}+o \cdot \varepsilon_{p} \cdot A_{\mathrm{H} 2 \mathrm{O}}
\end{aligned}
$$

Boudouard function:

$$
\begin{aligned}
\log _{10}\left(\frac{d X}{d t}\right)= & a+b \cdot T+c \cdot P_{\mathrm{CO} 2}+d \cdot e_{p}+e \cdot \varepsilon_{p}+f \cdot A_{\mathrm{CO} 2}+g \cdot T^{2} \\
& +h \cdot P_{\mathrm{CO} 2}^{2}+i \cdot T \cdot e_{p}+j \cdot T \cdot \varepsilon_{p}+K \cdot T \cdot A_{\mathrm{CO} 2} \\
& +l \cdot e_{p} \cdot \varepsilon_{p}+m \cdot e_{p} \cdot A_{\mathrm{CO} 2}+n \cdot \varepsilon_{p} \cdot A_{\mathrm{CO} 2}
\end{aligned}
$$

Combustion function: 


$$
\begin{aligned}
\log _{10}\left(\frac{d X}{d t}\right)= & a+b \cdot T+c \cdot P_{02}+d \cdot e_{p}+e \cdot \varepsilon_{p}+f \cdot A_{02}+g \cdot T^{2} \\
& +h \cdot P_{02}^{2}+i \cdot e_{p}^{2}+j \cdot \varepsilon_{p}^{2}+K \cdot T \cdot P_{02}+l \cdot T \cdot e_{p} \\
& +m \cdot T \cdot A_{02}
\end{aligned}
$$

The accuracy of these functions in predicting particle conversion rate is illustrated in Figs. 4-6 for steam gasification, Boudouard, and oxidation reactions respectively. In these plots, we compare the particle conversion rates calculated by the function to the one given by the particle model (numerical data). Moreover, we added the experimental data used for particle model calibration in the plots. In any of the three figures, each graph represents the response of the particle conversion rate to the variation in only one parameter, the values of the other parameters being set at referential conditions (Section 4.2). The development domain in which the functions were calculated is also mentioned in the graphs. These

\section{Table 4}

Correlation coefficient $R^{2}$ and predictive coefficient $Q^{2}$ of apparent reactivity functions.

\begin{tabular}{llll}
\hline & Steam gasification & Boudouard & Combustion \\
\hline$R^{2}(-)$ & 0.998 & 0.996 & 0.984 \\
$Q^{2}(-)$ & 0.989 & 0.980 & 0.953 \\
\hline
\end{tabular}

figures show also the behavior of the functions outside the development domain.

In the development domain, these figures show that the functions very accurately predict the numerical results for the five parameters. Differences were observed in several cases and are discussed below.

Fig. 4 shows results obtained for steam gasification. In the development domain, the coefficient of variation (CV) - defined as the ratio of the standard deviation to the mean between function and model results - is less than $6 \%$. This low value is quite acceptable when the aim is predicting particle conversion rates given the complexity of the mechanism involved and the uncertainties in experimental measurements. Functions outside the validity domain cannot be applied as they are not validated.

Concerning the Boudouard reaction (Fig. 5) the coefficient of variation between the model and the function was less than $6 \%$, which is quite acceptable.

Concerning the combustion reaction (Fig. 6) the accuracy of the function was slightly lower than the two other reactions: the coefficient of variation was less than $10 \%$ in the development domain. This uncertainty is nevertheless acceptable. Let us recall that this is more or less the accuracy of the experimental results that were used to calibrate the particle model [10]. Fig. 6e shows that the particle conversion rate calculated by the particle model increases
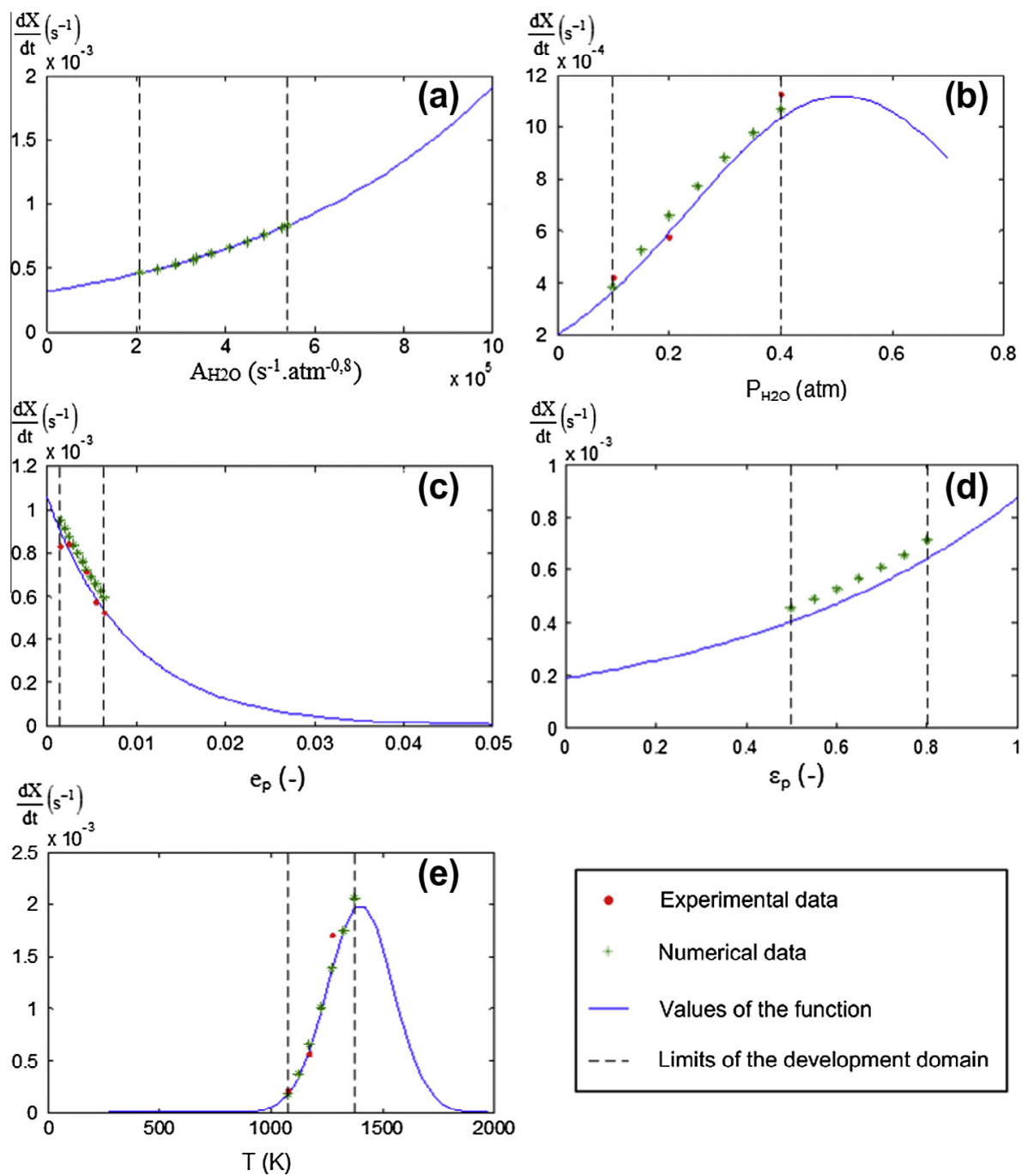

Fig. 4. Validation of the apparent reactivity function for steam gasification reaction. 

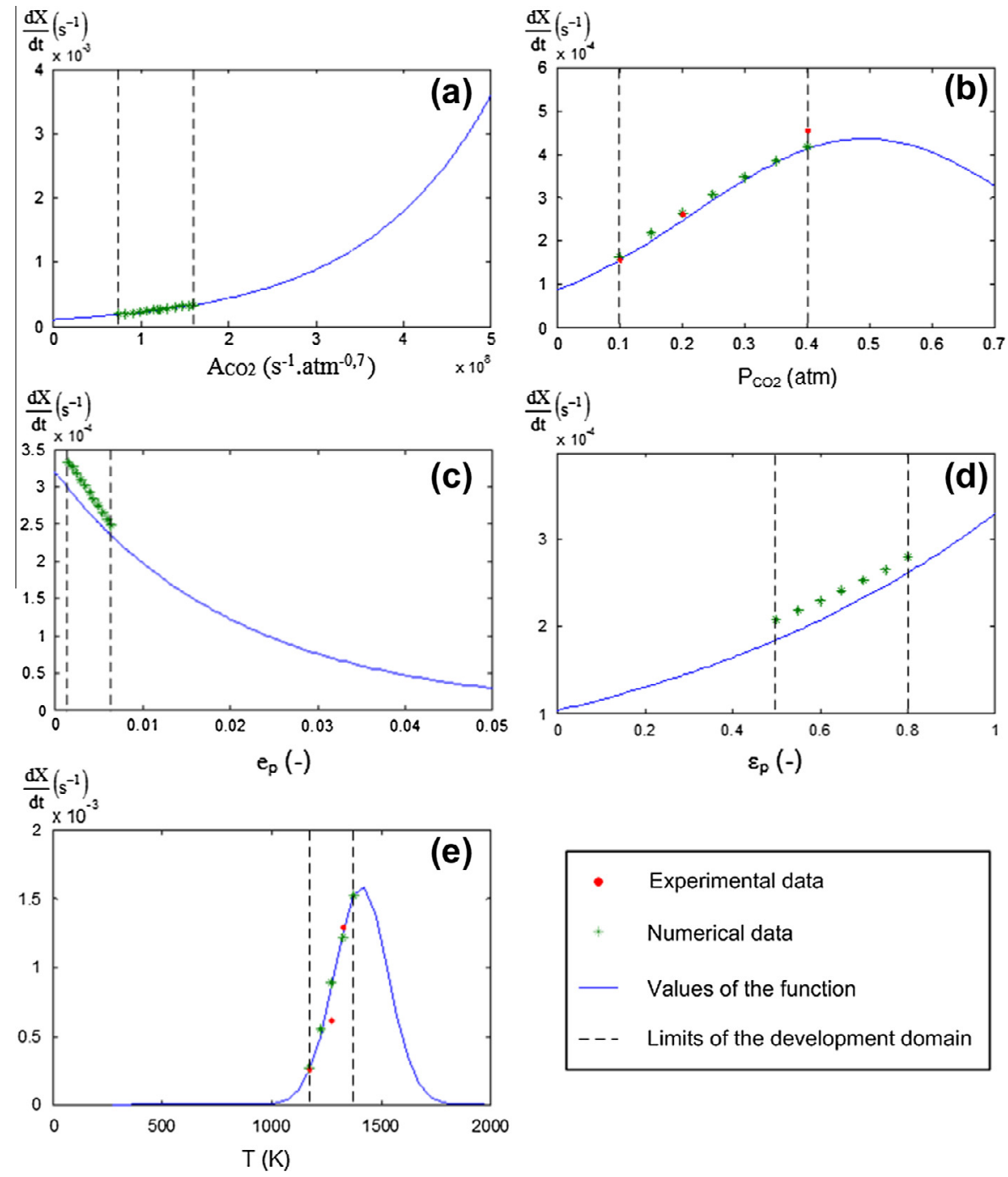

Fig. 5. Validation of the apparent reactivity function for the Boudouard reaction.

with temperature between 1000 and $1200 \mathrm{~K}$. Above, it remains constant. This can be explained by a change in the particle conversion regime: above $1200 \mathrm{~K}$, conversion is limited by external mass transfer and no longer depends on temperature. This change of behavior in the conversion regime is difficult to capture by the function. In Fig. 6a, we observe that the particle conversion rate calculated with the particle model is not sensitive to pre-exponential factor (Section 4.2). This behavior is incorrectly displayed by the function which fails to reproduce sensitivity to this parameter $A$. Indeed, the low impact of " $A$ " on apparent reactivity makes it difficult for the experimental design to take into account the influence of this parameter.

\section{Adaptation to char made of wood pellets}

In previous sections we validated the functions for particles of wood chip char in $\mathrm{N}_{2}$ atmospheres containing $\mathrm{H}_{2} \mathrm{O}, \mathrm{CO}_{2}$ and $\mathrm{O}_{2}$. When a new char is considered, the previous functions are not useable as is. Properties such as porosity $\left(\varepsilon_{0}\right)$ and pre-exponential factor $\left(A_{j}\right)$ have to be updated in the function. Initial porosity can be easily measured in the laboratory. Determination of the pre-exponential factor is more complex and requires additional experiments.
The new char considered here is a wood pellet char (WPC). Table 5 compares the properties of the two chars. Porosity is the major difference between WCC and WPC: WCC porosity is $40 \%$ lower than WPC porosity.

\subsection{Experiments on wood pellet char}

Regarding the determination of $A_{i}$, we used the "Macro-TG" reactor to carry out the experiments $[3,10,19,20]$. In theory, only one experiment is needed in each atmosphere. Nevertheless, we conducted several experiments in each reactive atmosphere $\left(\mathrm{H}_{2} \mathrm{O} / \mathrm{N}_{2}, \mathrm{CO}_{2} / \mathrm{N}_{2}\right.$ and $\left.\mathrm{O}_{2} / \mathrm{N}_{2}\right)$, each time varying partial pressure $p_{j}$ or temperature $T$ (Table 6 ). This ensured that the determination of $A_{i}$ was more accurate and above all that $A_{i}$ was validated in a wider range of operating conditions.

In Fig. 7, the conversion ratio is plotted against time for the new WPC. The particle conversion rate of WPC, given by the slope of the curves, remained more or less constant until $90 \%$ of char conversion was completed and decreased thereafter, as already observed in the case of wood chips char.

Steam gasification (Fig. 7a) is very sensitive to temperature: complete conversion was reached after 1140,2340 , and $7320 \mathrm{~s}$ at temperatures of 1273,1173 , and $1073 \mathrm{~K}$ respectively. That is to 

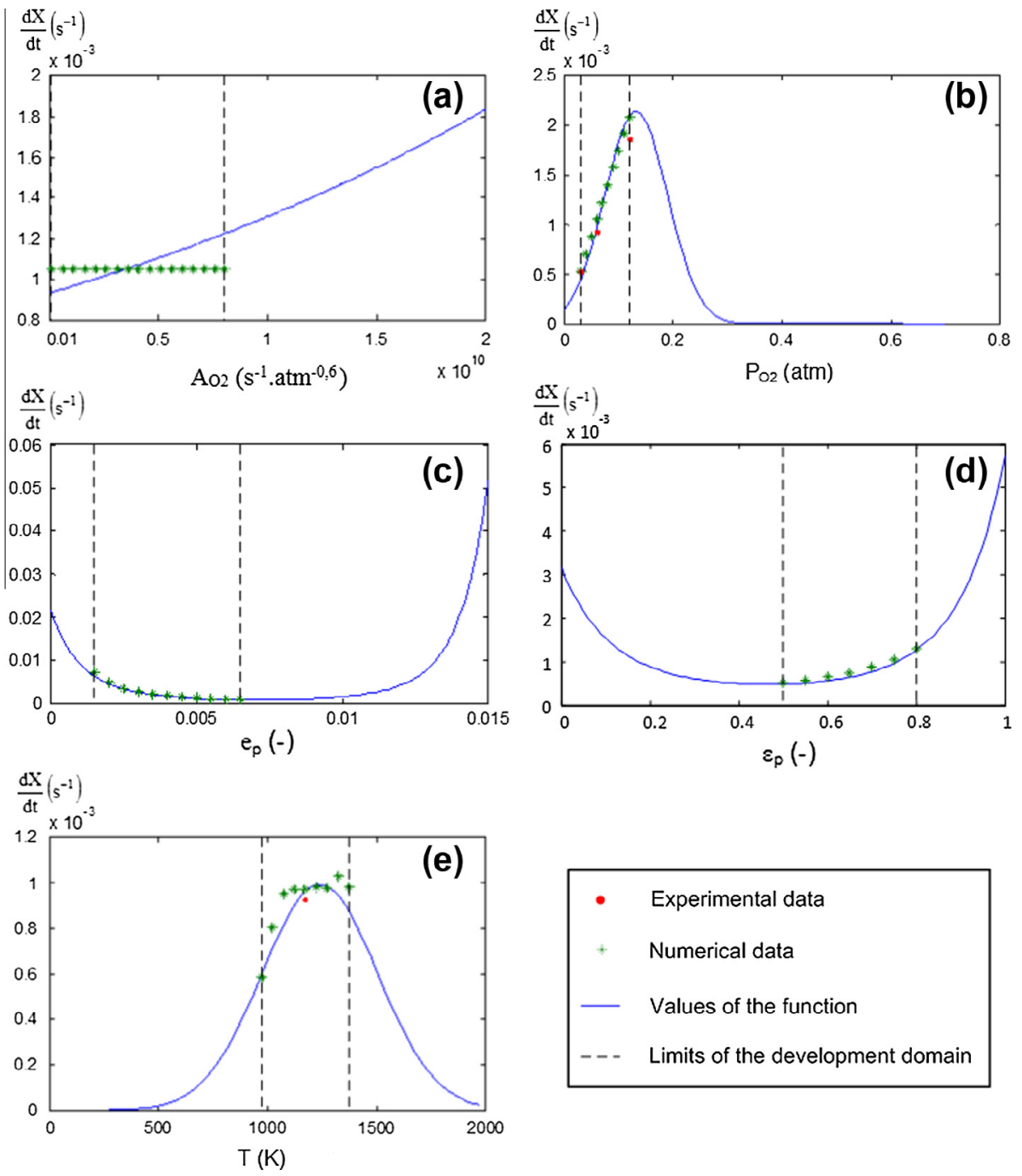

Fig. 6. Validation of the apparent reactivity function for the oxidation reaction.

Table 5

WCC and WPC properties.

\begin{tabular}{lll}
\hline & WCC & WPC \\
\hline$e_{p, 0}(\mathrm{~mm})$ & $1.5-6.5$ & 4.2 \\
$\varepsilon_{0}(-)$ & 0.74 & 0.51 \\
$A_{\mathrm{H} 2 \mathrm{O}}\left(\mathrm{s}^{-1} \mathrm{~atm}^{-0.8}\right)$ & $3.55 \times 10^{5}$ & $2.80 \times 10^{5}$ \\
$A_{\mathrm{CO} 2}\left(\mathrm{~s}^{-1} \mathrm{~atm}^{-0.7}\right)$ & $1.20 \times 10^{8}$ & $1.06 \times 10^{8}$ \\
$A_{\mathrm{O} 2}\left(\mathrm{~s}^{-1} \mathrm{~atm}^{-0.6}\right)$ & $1.10 \times 10^{9}$ & $3.11 \times 10^{9}$ \\
\hline
\end{tabular}

say a $200 \mathrm{~K}$ increase in gasification temperature resulted in 6.5 times higher reactivity. At the reference temperature of $1173 \mathrm{~K}$, we varied the concentration of steam in the atmosphere. Gasification was completed at 2340 and $4260 \mathrm{~s}$ with 0.2 and 0.1 atm $\mathrm{H}_{2} \mathrm{O}$ (in $\mathrm{N}_{2}$ ), respectively. Steam gasification was thus about 3 times faster with 0.2 atm $\mathrm{H}_{2} \mathrm{O}$ than with 0.1 atm $\mathrm{H}_{2} \mathrm{O}$.

The same trend was observed for gasification with carbon dioxide (Fig. 7b). Gasification was completed after 1860 and $4320 \mathrm{~s}$ at temperatures of 1273 and $1173 \mathrm{~K}$ respectively: the particle conversion rate was 2.3 times faster. Complete conversion was reached after $4320 \mathrm{~s}$ with $0.2 \mathrm{~atm} \mathrm{CO}_{2}$ while it was reached only after 3000 s with 0.2 atmCO $_{2}$.

During combustion (Fig. 7c), the particle conversion rate is sensitive to $\mathrm{O}_{2}$ partial pressure: it was 4 times faster at $0.12 \mathrm{~atm} \mathrm{O}_{2}$ than at $0.03 \mathrm{~atm} \mathrm{O}_{2}$ with complete conversion after 960 and
Table 6

Operating conditions for WPC gasification experiments.

\begin{tabular}{lll}
\hline Reactant gas & Partial pressure (atm) & Temperature (K) \\
\hline $\mathrm{H}_{2} \mathrm{O}$ & 0.1 & \\
& 0.2 & 1173 \\
& 0.4 & 1073 \\
& 0.2 & 1273 \\
& 0.2 & \\
$\mathrm{CO}_{2}$ & 0.2 & 1173 \\
& 0.4 & 1273 \\
$\mathrm{O}_{2}$ & 0.2 & 1173 \\
& 0.03 & 1273 \\
\end{tabular}

$3900 \mathrm{~s}$ respectively. However, the impact of temperature was slight: conversion was complete after 3180 and $3900 \mathrm{~s}$ at temperatures of 1273 and $1173 \mathrm{~K}$ respectively.

\subsection{Determination of $A_{i}$}

The determination of $A_{i}$ is simple and does not require running the statistical method again. This is one advantage of the present functions. First, we calculated the particle conversion rate for each 

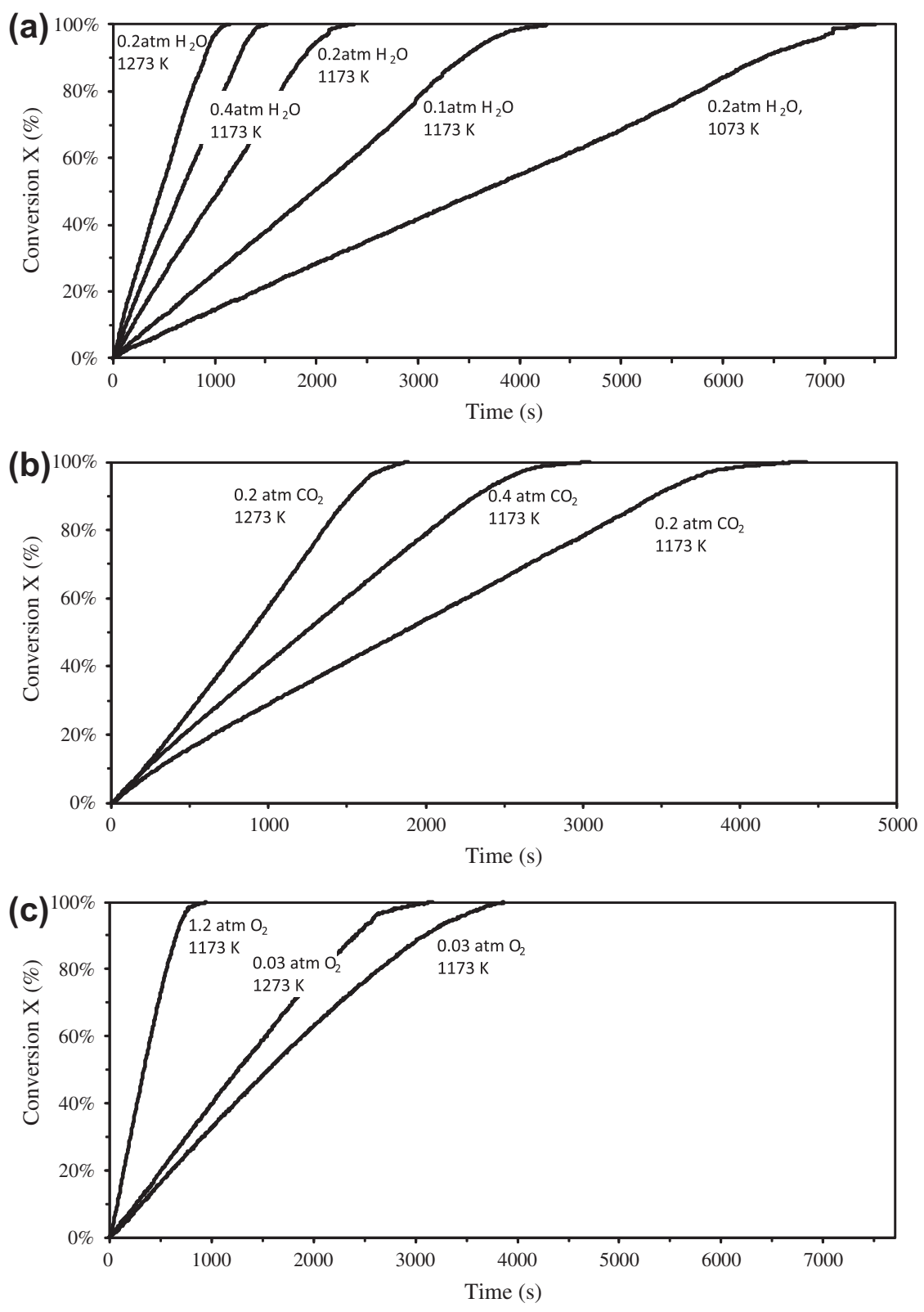

Fig. 7. Results of WPC gasification under $\mathrm{H}_{2} \mathrm{O} / \mathrm{N}_{2}(\mathrm{a}), \mathrm{CO}_{2} / \mathrm{N}_{2}$ (b) and $\mathrm{O}_{2} / \mathrm{N}_{2}$ (c) atmosphere.

Table 7

Comparison of experimental and calculated apparent reactivity case of steam gasification.

\begin{tabular}{llllll}
\hline$T(\mathrm{~K})$ & $\mathrm{H}_{2} \mathrm{O}(\mathrm{atm})$ & $\left(\frac{d X}{d t}\right)_{\exp }^{50}$ & $\left(\frac{d X}{d t}\right)_{\text {calc }}^{50}$ & (Exp - calc) & $\mathrm{CV}(\%)$ \\
\hline 1173 & 0.1 & $2.53 \times 10^{-4}$ & $2.89 \times 10^{-4}$ & $1.30 \times 10^{-9}$ & 6.63 \\
1173 & 0.2 & $4.81 \times 10^{-4}$ & $4.59 \times 10^{-4}$ & $4.63 \times 10^{-10}$ & 2.29 \\
1173 & 0.4 & $7.49 \times 10^{-4}$ & $7.77 \times 10^{-4}$ & $7.66 \times 10^{-10}$ & 1.81 \\
1073 & 0.2 & $1.38 \times 10^{-4}$ & $1.27 \times 10^{-4}$ & $1.28 \times 10^{-10}$ & 4.27 \\
1273 & 0.2 & $1.07 \times 10^{-3}$ & $1.04 \times 10^{-3}$ & $1.17 \times 10^{-9}$ & 1.62 \\
\hline
\end{tabular}

experiment (Table 7 , third column). The operating conditions ( $T$, $\left.P_{\mathrm{H} 2 \mathrm{O}}\right)$ and char properties $\left(e_{p, 0}, \varepsilon_{0}\right)$ were known. So, we adjusted $A_{\mathrm{H} 2 \mathrm{O}}$ by minimizing the sum of the squares of the deviations (5th column) between the particle conversion rate calculated by the function from Eq. (9) (4th column) and the one determined experimentally (third column). The value determined was $A=2.8 \times 10^{5} \mathrm{~s}^{-1} \mathrm{~atm}^{-0.8}$ in the case of steam gasification.

\subsection{Validation of $A_{i}$}

In Fig. 8, we compare $A_{i}$ for WCC and WPC for the three reactions. It is important to note that that new values of $A_{i}$ (WPC) are in the range of $A_{i}$ used for calculating the function (Table 2). For steam gasification and for the Boudouard reaction, differences between WCC and WPC were small. These results are satisfactory since WWC and WPC were both produced from maritime pine. Moreover the two initial chars were produced in the same pyrolysis operating conditions (temperature $750 \mathrm{~K}$, residence time $1 \mathrm{~h}$; flow rate $15 \mathrm{~kg} \mathrm{~h}^{-1}$ ) [14].

Concerning combustion, $A_{i}$ was 3 times higher for WCC than for WPC. But as already mentioned in Section 3, in the case of combustion, the function fails to apprehend the sensitivity of the particle conversion rate to pre-exponential factor.

Finally, in Fig. 9, we present the comparison between experimental particle conversion rates and those calculated with the functions in the three atmospheres. Concerning steam gasification and combustion, the 


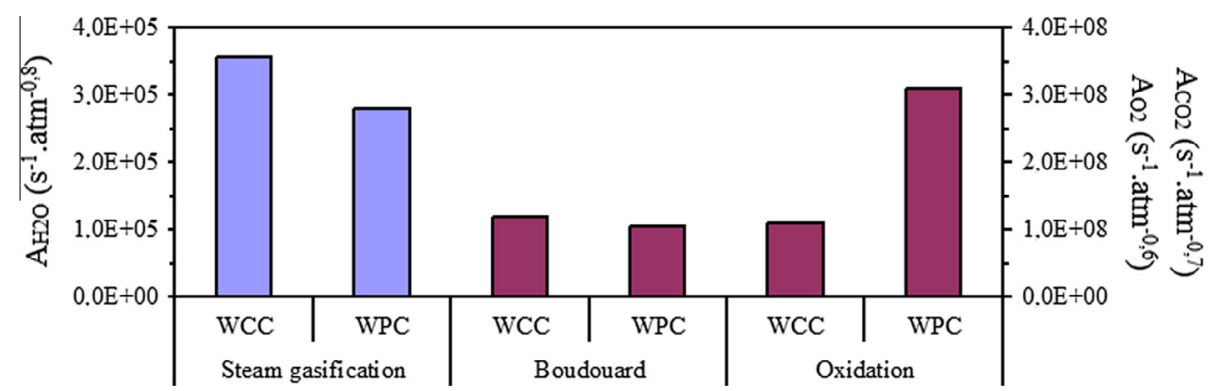

Fig. 8. WCC and WPC pre-exponential factors for the three reactions.

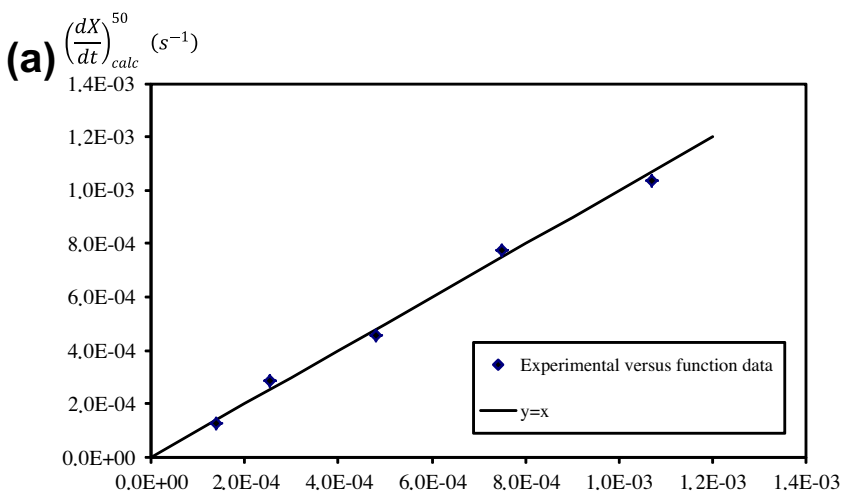

(b)

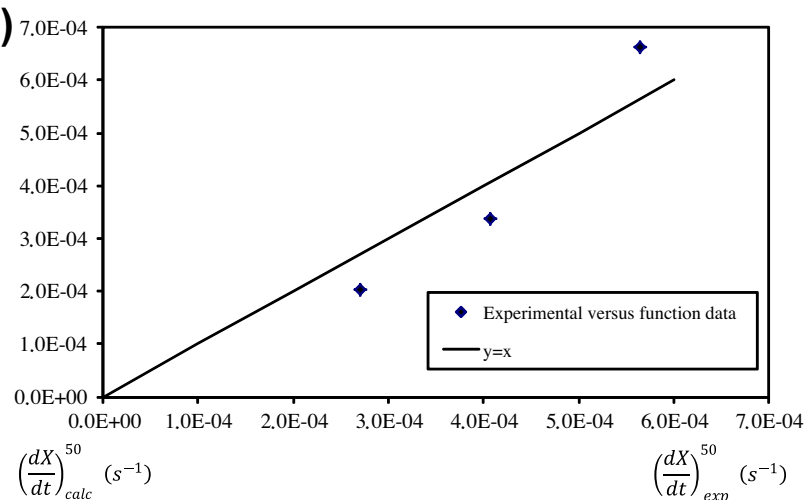

(c)

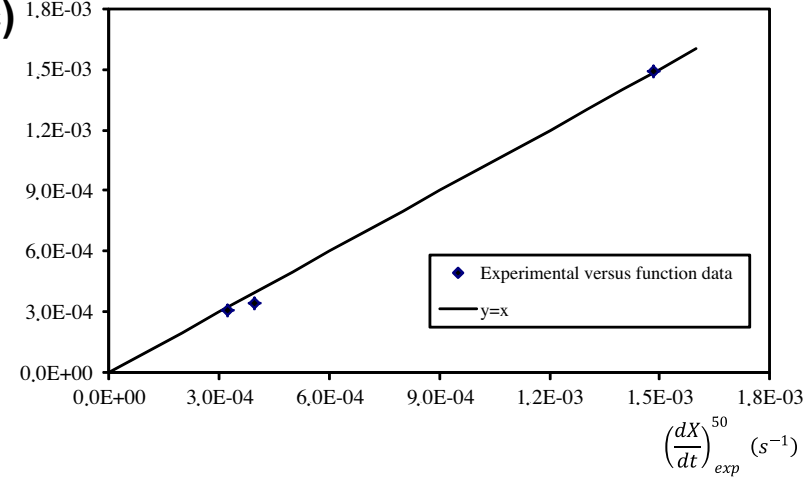

Fig. 9. Comparison of apparent reactivities: experimental versus function data.

coefficient of variation was less than $7 \%$. For the Boudouard reaction, the coefficient of variation was less than $14 \%$. Nevertheless given the complexity of the phenomena and the uncertainties on the experiments [10] these results are quite acceptable. In conclusion, the particle conversion rate functions determined for wood chips char can be used for wood pellets char with the new calculated pre-exponential factors.

\section{Conclusion}

Three functions were established to calculate the particle conversion rate of steam gasification, Boudouard reaction and combustion for wood char chips. These functions take into account the phenomena that control the particle conversion rate of a char particle under a reactive atmosphere, namely external and internal heat/mass transfers and intrinsic reaction kinetics. The functions depend on the operating conditions selected - temperature $T$, partial pressure of the reactant gas $p_{i}-$ and on selected char properties: porosity $\varepsilon_{0}$, particle thickness $e_{p, 0}$ and reaction preexponential factor $A_{i}$.

The determination is based on an original method using a particle model and an experimental design.

A function $\frac{d X}{d t}=f\left(T, P_{i}, \varepsilon_{p, 0}, e_{p, 0}, A_{i}\right)$ was determined for each reactant gas: $\mathrm{H}_{2} \mathrm{O}, \mathrm{CO}_{2}$ and $\mathrm{O}_{2}$.

Using the example of wood pellet char, we showed then that these functions can be easily adapted to a new wood char provided that experimental results are in good agreement with function predictions during pre-exponential factors determination step. The later was performed by confronting 3-5 experimental results to the functions. Additional studies are now necessary to show the functions adaptability to a char from a new biomass nature.

These functions can be used to express the source terms in the heat and mass balance equations of academic or industrial codes of gasifiers, provided that operating conditions and char properties are within the validity domains of the functions.

\section{References}

[1] Dasappa S, Paul PJ, Mukunda HS, Shrinivasa U. Wood-char gasification: experiments and analysis on single particles and packed beds 1998:1335-42.

[2] Gomez-Barea A, Ollero P, Villanueva A. Diffusional effects in $\mathrm{CO}_{2}$ gasification experiments with single biomass char particles. 2. Theoretical predictions. Energy Fuels 2006;20(5):2211-22.

[3] Mermoud F, Golfier F, Salvador S, Van de Steene L, Dirion JL. Experimental and numerical study of steam gasification of a single charcoal particle. Combust Flame 2006:145(1-2):59-79.

[4] Golfier F, Van de Steene L, Salvador S, Mermoud F, Oltean C, Bues MA. Impact of peripheral fragmentation on the steam gasification of an isolated wood charcoal particle in a diffusion-controlled regime. Fuel 2009;88(8):1498-503.

[5] Di Blasi C. Dynamic behaviour of stratified downdraft gasifiers. Chem Eng Sci 2000;55(15):2931-44.

[6] Di Blasi C. Modeling wood gasification in a countercurrent fixed-bed reactor. AIChE J 2004;50(9):2306-19.

[7] Hobbs ML, Radulovic PT, Smoot LD. Modeling fixed-bed coal gasifiers. AIChE J 1992.

[8] Mandl C, Obernberger I, Biedermann F. Modelling of an updraft fixed-bed gasifier operated with softwood pellets. Fuel 2010;89(12):3795-806.

[9] Radulovic PT, Ghani MU, Smoot LD. An improved model for fixed bed coal combustion and gasification. Fuel 1995;74(4):582-94.

[10] Van de Steene L, Tagutchou JP, Escudero Sanz FJ, Salvador S. Gasification of woodchip particles: experimental and numerical study of char- $\mathrm{H}_{2} \mathrm{O}$, char- $\mathrm{CO}_{2}$, and char-O2 reactions. Chem Eng Sci 2011;66(20):4499-509.

[11] Liliedahl T, Sjöström K. Modelling of char-gas reaction kinetics. Fuel 1997;76(1):29-37.

[12] Muhlen HJ, Van Heek KH, Juntgen H. Kinetic studies of steam gasification of char in the presence of $\mathrm{H}_{2}, \mathrm{CO}_{2}$ and CO. Fuel 1985;64:944-9. 
[13] Mermoud F. Gazéification de charbon de bois à la vapeur d'eau: de la particule isolée au lit fixe continu [Thèse de Doctorat]. Institut Polytechnique de Toulouse; 2006.

[14] Teixeira G, Van de Steene L, Martin E, Gelix F, Salvador S. Gasification of char from wood pellets and from wood chips: textural properties and thermochemical conversion along a continuous fixed bed. Fuel 2012:102:514-24

[15] Kirubakaran V, Sivaramakrishnan V, Nalini R, Sekar T, Premalatha M Subramanian P. A review on gasification of biomass. Renew Sustain Energy Rev 2009;13(1):179-86.

[16] Paviet F, Bals O, Antonini G. The effects of diffusional resistance on wood char gasification. Process Safe Environ Protect 2008;86(2):131-40.
[17] Struis RPWJ, von Scala C, Stucki S, Prins R. Gasification reactivity of charcoal with $\mathrm{CO}_{2}$. Part II: metal catalysis as a function of conversion. Chem Eng Sci 2002;57(17):3593-602.

[18] Struis RPWJ, von Scala C, Stucki S, Prins R. Gasification reactivity of charcoal with $\mathrm{CO}_{2}$. Part I: conversion and structural phenomena. Chem Eng $\mathrm{Sc}$ 2002;57(17):3581-92.

[19] Mermoud F, Salvador S, Van de Steene L, Golfier F. Influence of the pyrolysis heating rate on the steam gasification rate of large wood char particles. Fuel 2006;85(10-11):1473-82.

[20] Van de Steene L, Salvador S, Charnay G. Controlling powdered fuel combustion at low temperature in a new entrained flow reactor. Combust Sci Technol 2000;159(1):255-79. 